\title{
SNP Microarray in FISH Negative Clinically Suspected 22q11.2 Microdeletion Syndrome
}

\author{
Ashutosh Halder, Manish Jain, and Amanpreet Kaur Kalsi \\ Reproductive Biology, AIIMS, New Delhi 110029, India \\ Correspondence should be addressed to Ashutosh Halder; ashutoshhalder@gmail.com
}

Received 28 December 2015; Revised 27 January 2016; Accepted 16 February 2016

Academic Editor: Albert Basson

Copyright ( 2016 Ashutosh Halder et al. This is an open access article distributed under the Creative Commons Attribution License, which permits unrestricted use, distribution, and reproduction in any medium, provided the original work is properly cited.

The present study evaluated the role of SNP microarray in 101 cases of clinically suspected FISH negative (noninformative/normal) 22q11.2 microdeletion syndrome. SNP microarray was carried out using $300 \mathrm{~K}$ HumanCytoSNP-12 BeadChip array or CytoScan $750 \mathrm{~K}$ array. SNP microarray identified 8 cases of 22q11.2 microdeletions and/or microduplications in addition to cases of chromosomal abnormalities and other pathogenic/likely pathogenic CNVs. Clinically suspected specific deletions (22q11.2) were detectable in approximately $8 \%$ of cases by SNP microarray, mostly from FISH noninformative cases. This study also identified several LOH/AOH loci with known and well-defined UPD (uniparental disomy) disorders. In conclusion, this study suggests more strict clinical criteria for FISH analysis. However, if clinical criteria are few or doubtful, in particular newborn/neonate in intensive care, SNP microarray should be the first screening test to be ordered. FISH is ideal test for detecting mosaicism, screening family members, and prenatal diagnosis in proven families.

\section{Introduction}

The 22q11.2 microdeletion syndrome is the most common microdeletion syndrome and seen at a prevalence of 1 in 4000 to 6000 live births [1]. It is characterized by hemizygous microdeletion of $\leq 3 \mathrm{mb}$ size of chromosome 22q11.2 locus in which several genes are lost. It is mostly spontaneous/de novo and in $<10 \%$ cases are inherited [2-4]. It is frequently associated with multiple congenital anomalies, in particular cardiac anomaly (conotruncal cardiac anomaly such as Fallot's tetralogy, interrupted aortic arch, truncus arteriosus, or major aortopulmonary collateral), developmental delay, hypocalcaemia, immune deficiency, cleft palate or velopharyngeal insufficiency or swallowing difficulty, and dysmorphism (broad bulbous nose, square shaped tip of nose, short philtrum, telecanthus, slanting eyes, low set ears, etc.). FISH, until recent, was commonly used for precise genetic diagnosis of common microdeletion syndromes, including 22q11.2 microdeletion [5-8]. However, FISH provides information only on targeted locations and does not allow a comprehensive evaluation of the whole genome. In addition, atypical smaller deletions are difficult to identify by FISH due to failure in covering those locations by single FISH probe (outside the region of hybridisation by the FISH probe). Furthermore, it is difficult to detect 22q11.2 duplication by FISH due to variable size of signals as well as distraction with split signals of normal cells. Often 22q11.2 duplication displays features like 22q11.2 deletion syndrome [9]. Hence, FISH alone cannot provide reliable diagnosis for cases of 22q11.2 microdeletions/duplications syndrome. Further, if FISH is used for doubtful cases (if few numbers of clinical criteria are fulfilled by patient) then chances of detecting targeted deletion are less frequent $[7,8]$. High-resolution array CGH was used to investigate FISH negative for 22q11.2 deletions cases with conotruncal heart defects $[10]$ and additional cases of 22q11.2 microdeletion/microduplication containing TBX1 gene were detected. In this study with SNP microarray we have assessed 101 cases of FISH negative/noninformative clinically suspected 22q11.2 microdeletion syndrome to determine whether any benefit is gained from using SNP microarray. 


\section{Material and Methods}

This study was conducted in the Department of Reproduction Biology, All India Institute of Medical Sciences, New Delhi, India, from September 2011 to August 2014. A total of $101 \mathrm{FISH}$ negative/noninformative (FISH was normal in 85 cases, was not attempted in 12 cases due to frozen/clotted samples, and failed in 4 cases due to few/lysed/clumped cells) clinically suspected 22q11.2 microdeletion syndrome cases were prospectively enrolled for the study using SNP microarray. FISH was carried out using noncommercial FISH probe (22q11.2; RP5-882J5; genomic coordinate/exact position of probe is unavailable; obtained from Uniba Biologia, University of Bari, Italy, by curtsy of Professor Mariano Rocchi, http://www.biologia.uniba.it/rmc/). SNP microarray was used to detect chromosomal abnormality (aneuploidy, triploidy, mosaicism, etc.) as well as microdeletion, microduplication, UPD/LOH, and so forth as described before [11]. All cases were referred from various hospitals of Delhi (northern India) for confirmation of clinical diagnosis. The Institutional Human Ethics Committee approved research protocol. Most patients (excluding very sick/intensive care patients) underwent clinical genetics evaluation (directly by our team or by referral physician) as per specific 22q11.2 microdeletion syndrome proforma (Supplementary file 1 in Supplementary Material available online at http://dx.doi.org/10.1155/2016/5826431). About 2 mL EDTA blood sample was collected from the affected individual. SNP microarray study was carried out commercially either from Illumina (HumanCytoSNP-12 DNA Analysis Bead Chip Kit $300 \mathrm{~K}$ ) or Affymetrix (GeneChip Human Mapping $750 \mathrm{~K}$ ) platforms. Images were captured on iScan System or Cytoscan, respectively. Data were analyzed (primary) using Illumina's KaryoStudio/GenomeStudio and Affymetrix ChAS software. Resolution was set as $0.1 \mathrm{mb}$ for deletion, $0.5 \mathrm{mb}$ for gain, and $3 \mathrm{mb}$ for $\mathrm{LOH} / \mathrm{AOH}$. Secondary analysis was carried out using web data analysis resources like DECIPHER (GRCH37; version 9.4), OMIM, and UPD with cross reference to UCSC, NCBI, Ensembl, and DGV. Interpretation and reporting followed American College of Medical Genetics standards and guidelines for interpretation and reporting of postnatal constitutional copy number variants [12].

\section{Result}

A total of 101 DNA samples from FISH negative/noninformative clinically suspected 22q11.2 microdeletion syndrome were analyzed by SNP microarray successfully. Details of SNP microarray findings of all 101 FISH negative/noninformative samples are available as master table (Supplementary file 2). Out of 101 FISH negative for 22q11.2 microdeletion cases, SNP microarray detected several cases of chromosomal abnormalities (Table 1), 22q11.2 microdeletion/microduplication (Table 2), other microdeletion/microduplication cases (Table 3), and several cases of likely pathogenic CNVs (Table 4). SNP microarray also detected several cases of LOH/AOH of known UPD disorders (Table 5) and some more likely pathogenic UPD disorders (Table 6) in the study.
This SNP microarray study identified 6 cases of chromosomal abnormalities (Table 1). Clinical feature in brief is presented in Table 7 (Figure 1). The study also identified eight cases of 22q11.2 microdeletions (7)/microduplication (1), mostly from FISH noninformative cases and 2 smaller CNVs (outside scope of FISH probe). Clinical feature in brief is presented in Table 8. This study detected 4 cases of other pathogenic CNVs (Table 3) and several cases of likely pathogenic CNVs (Table 4), which were outside the scope of FISH. Clinical features in brief of these cases are presented in Tables 9 and 10 (Figure 2). Microarray analysis identified 9 cases of segmental $\mathrm{LOH} / \mathrm{AOH}$, those associated with known UPD disorders (Table 5). Clinical features in brief of these cases are presented in Table 11 (Figure 3).

\section{Discussion}

We have been investigating microdeletion syndrome using FISH since 2005. Our experience with FISH in microdeletion syndrome including 22q11.2 microdeletion syndrome (most common microdeletion syndrome) is unsatisfactory as FISH detects approximately $8 \%$ of cases of 22q11.2 microdeletions $[7,8]$. The reasons for low positive rate of 22q11.2 microdeletion syndrome using RP5-882J5 PAC clone (FISH probe for 22q11.2 locus) were poor clinical inclusion criteria (often single criteria) and poor quality of sample referral (clotted/frozen/clumped blood samples/cells leading to FISH failure or omission of FISH tests). In our experience $(8,11-$ 13), FISH probe derived from RP5-882J5 PAC clone identifies all typical deletions ( $\geq 2 \mathrm{mb}$ ); however, it is unable to detect atypical deletions of $\leq 0.5 \mathrm{mb}$ sizes (at proximal or distal LCRs). Number of diagnostic criteria fulfilled by our patients ranged from one to eight and most consistent referral criteria were broad nose, TOF, swallowing difficulty, and hypocalcaemia/convulsion. Patient often was referred to our laboratory for 22q11.2 FISH testing based on a single clinical feature, namely, CHD (TOF) or convulsion. These lead to lower deletion detection rate. Poirsier et al. [13] also observed poor clinical criteria for FISH test referral.

In this prospective study, SNP microarray was carried out in 101 FISH negative/noninformative clinically suspected 22q11.2 microdeletion syndrome cases to assess the role of SNP microarray in the evaluation of clinically suspected 22q11.2 microdeletion syndrome. There were 12 samples that were not processed for FISH due to freezing or clotting. Microarray was successfully carried out in all 101 cases. Chen et al. [10], like this study, investigated FISH negative 22q11.2 deletion cases using high-resolution array CGH. They have found better diagnostic sensitivity of array CGH over FISH in fetuses with cardiac abnormalities associated with deletion 22q11.2 and duplication 22q11.2 syndromes. In the present study we have also detected several cases of chromosomal abnormalities (trisomy, triploidy, partial monosomy, or partial trisomy) and other pathogenic/likely pathogenic CNVs (Tables 1, 3, and 4). We have also picked up some smaller specific deletions/duplications outside the region of hybridisation of the FISH probe. Clinically suspected specific CNV (22q11.2) was detectable in approximately $8 \%$ of cases by SNP microarray over and above 


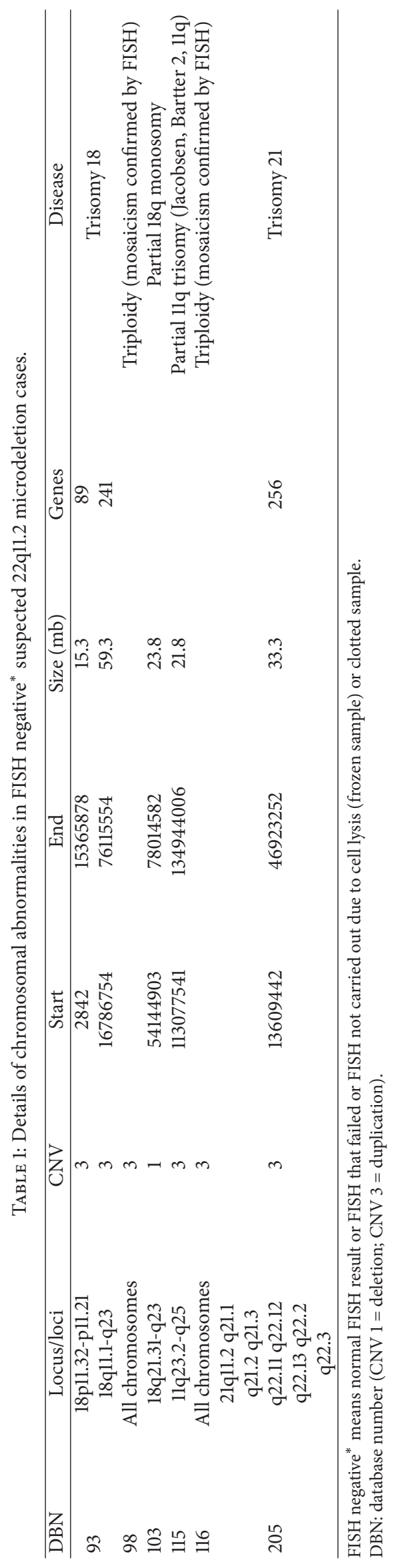




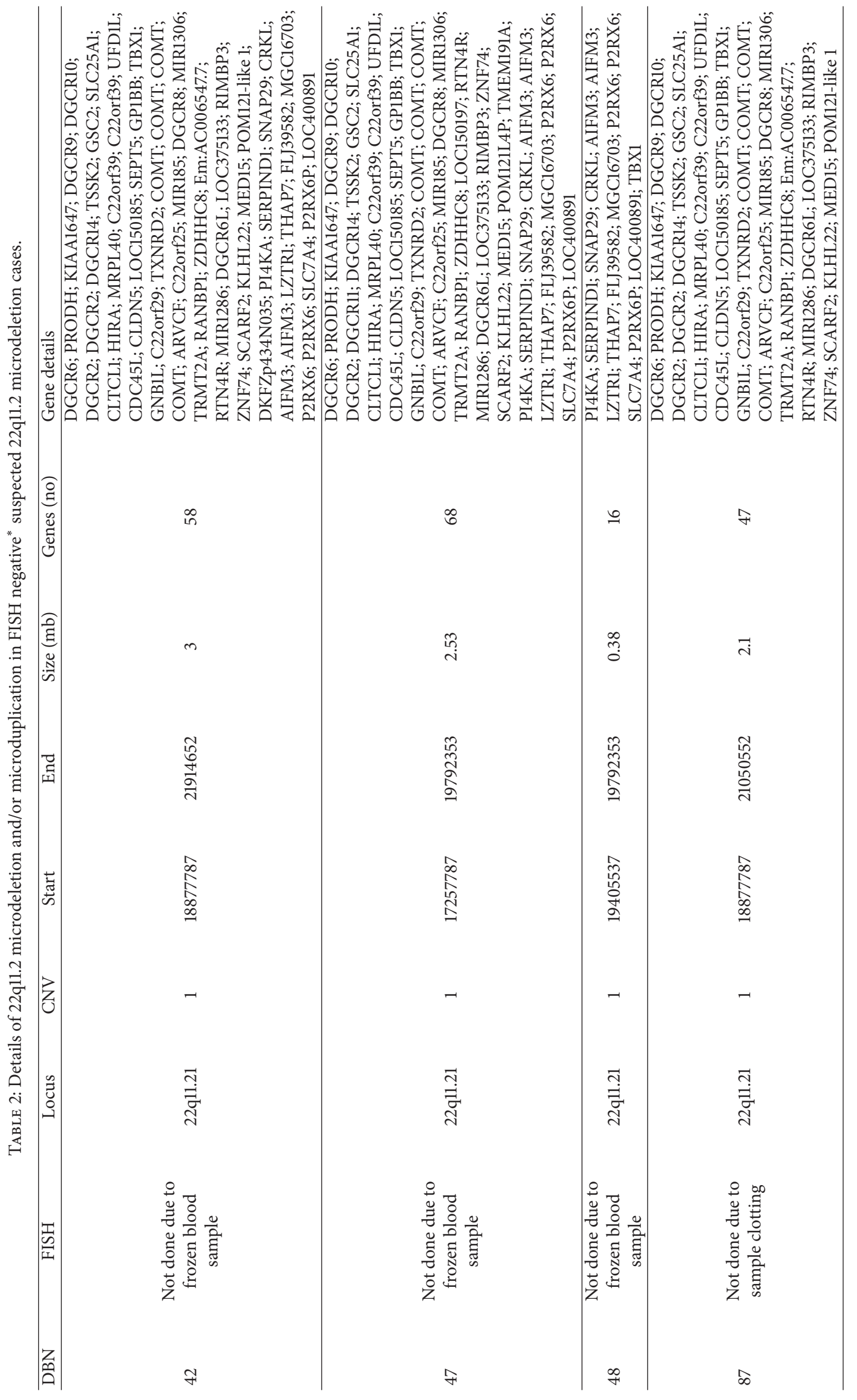




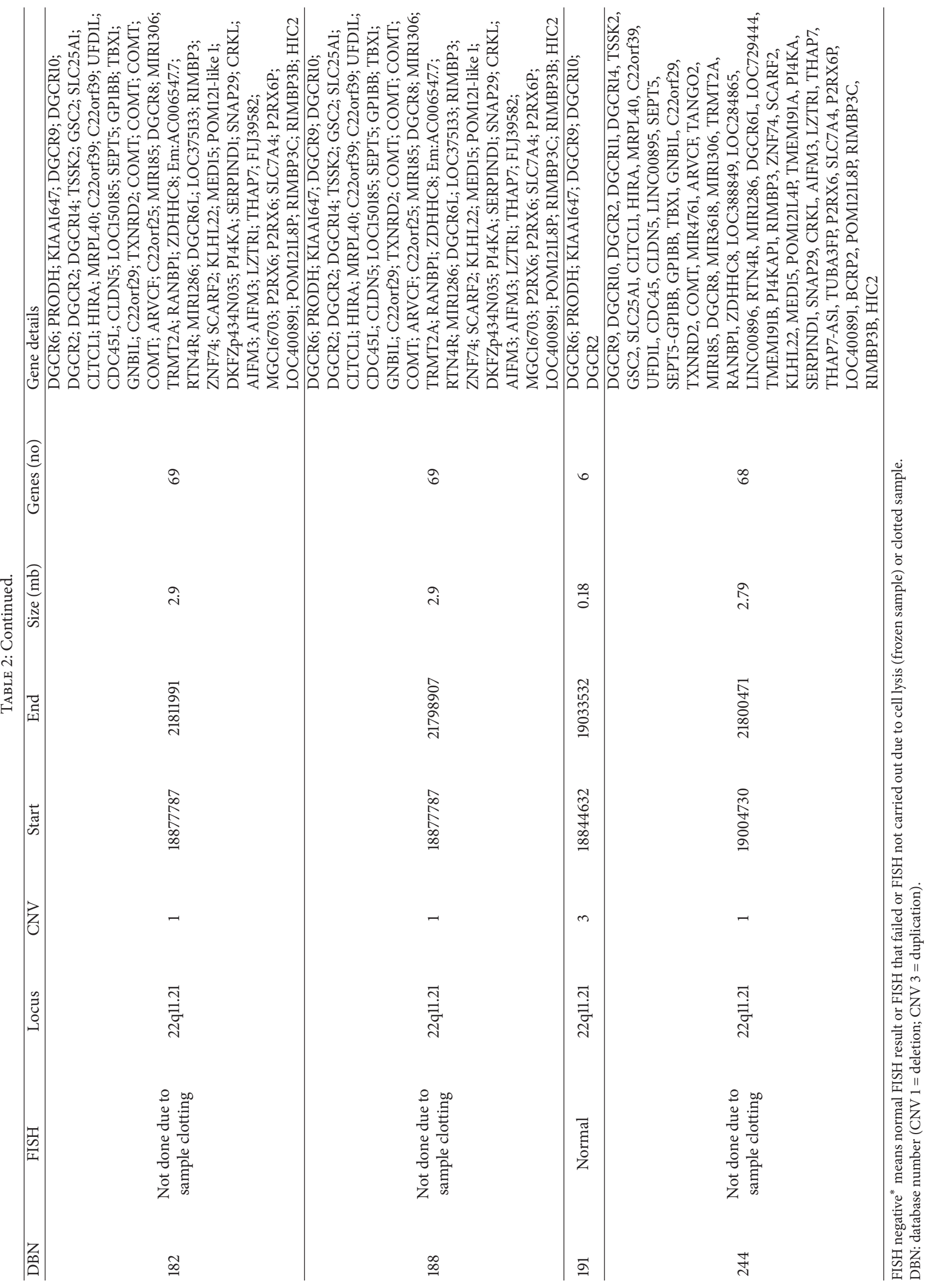




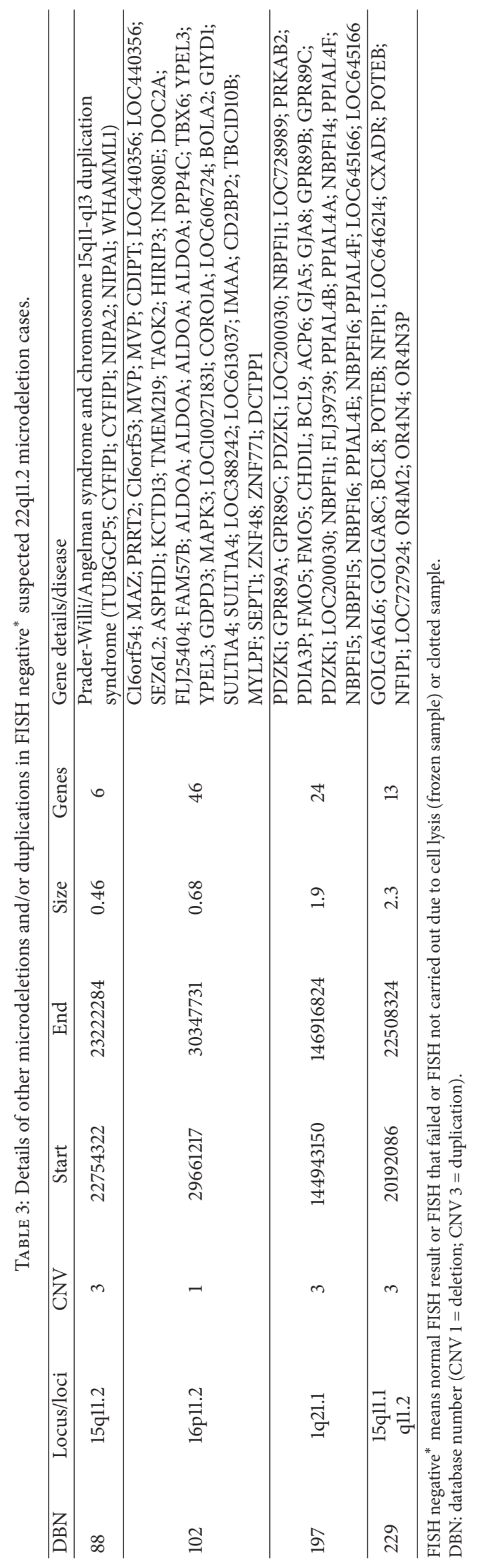




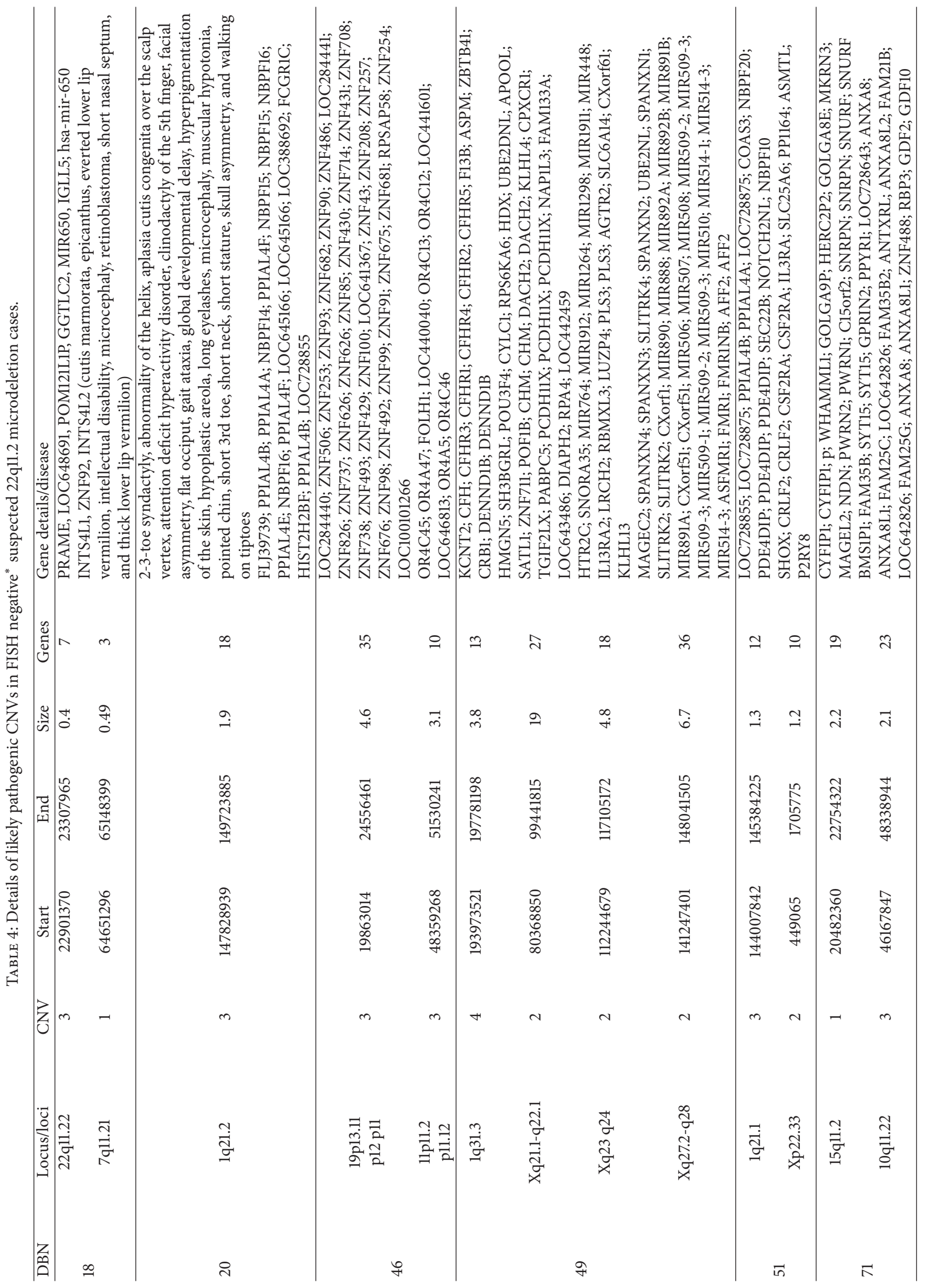




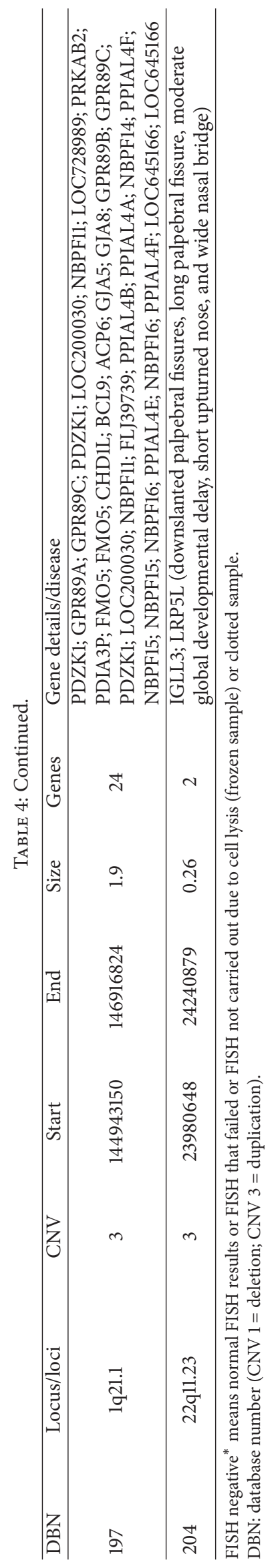




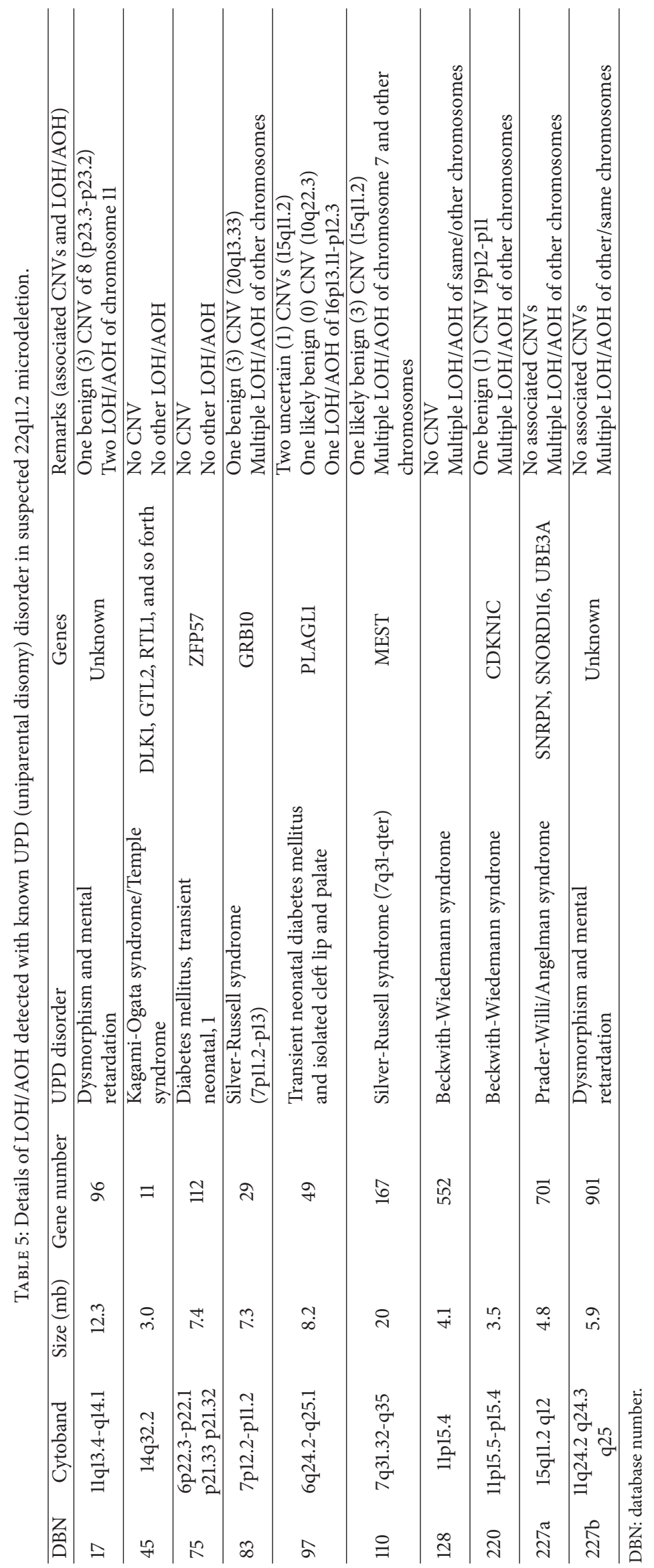


TABLE 6: Large (>5 mb) UPD/LOH/AOH locus/loci detected in suspected 22q11.2 microdeletion* .

\begin{tabular}{|c|c|c|c|c|}
\hline DBN & Cytoband & Size $(\mathrm{mb})$ & Gene number & Remarks (associated CNVs and LOH/AOH) \\
\hline 20 & $6 \mathrm{p} 21.1 \mathrm{p} 12.3$ & 5.7 & 38 & $\begin{array}{l}\text { One uncertain (3) CNV of chromosome } 1 \text { (q21.2) } \\
\text { Multiple LOH/AOH of chromosomes } 6 \text { and } 1\end{array}$ \\
\hline 43 & $4 q 21.23-q 22.1$ & 8.7 & 38 & $\begin{array}{l}\text { One benign (1) CNV of chromosome } 9 \text { (p24.3) } \\
\text { One LOH/AOH of chromosome } 15\end{array}$ \\
\hline \multirow[t]{2}{*}{49} & $\begin{array}{l}\text { Xq21.1 q21.2 q21.31 } \\
\text { q21.32 q21.33 q22.1 }\end{array}$ & 19 & 28 & $\begin{array}{l}\text { Partial tetrasomy of chromosomes } 1-8,11-14 \text {, and } 18 \\
\text { and partial disomy of chromosome X }\end{array}$ \\
\hline & Xq27.2 q27.3 q28 & 6.7 & 25 & $\begin{array}{l}\text { Multiple } \mathrm{LOH} / \mathrm{AOH} \text { of chromosome } \mathrm{X} \text { and one } \\
\mathrm{LOH} / \mathrm{AOH} \text { of chromosome } 1\end{array}$ \\
\hline \multirow{15}{*}{73} & $2 \mathrm{p} 25.2 \mathrm{p} 25.1$ & 5.7 & 33 & \multirow{15}{*}{$\begin{array}{l}\text { One likely benign (3) CNV of chromosome } 4 \text { (q22.3) } \\
\text { Multiple LOH/AOH of many chromosomes }\end{array}$} \\
\hline & $2 q 21.1-q 23.3$ & 18 & 44 & \\
\hline & 3p21.31-p14.2 & 13 & 161 & \\
\hline & $3 q 21.1-3 q 23$ & 16 & 135 & \\
\hline & $4 \mathrm{q} 31.3-\mathrm{q} 35.1$ & 32 & 112 & \\
\hline & $6 \mathrm{p} 21.1 \mathrm{p} 12.3$ & 5.7 & 86 & \\
\hline & $9 \mathrm{p} 24.2-\mathrm{p} 23$ & 8.3 & 29 & \\
\hline & $10 \mathrm{q} 25.1-\mathrm{q} 26.11$ & 11.8 & 60 & \\
\hline & $10 \mathrm{q} 26.11-\mathrm{q} 26.3$ & 12.8 & 68 & \\
\hline & 11p15.4-p15.1 & 11.7 & 102 & \\
\hline & $14 \mathrm{q} 11.2 \mathrm{q} 12$ & 8.8 & 63 & \\
\hline & $14 q 12-q 22.3$ & 23.9 & 112 & \\
\hline & $15 \mathrm{q} 22.33-\mathrm{q} 25.2$ & 14.4 & 155 & \\
\hline & 18p11.31-p11.21 & 11.9 & 55 & \\
\hline & $18 \mathrm{q} 11.1-\mathrm{q} 12.3$ & 22.8 & 85 & \\
\hline 76 & $17 \mathrm{q} 12-\mathrm{q} 21.1$ & 5.3 & 102 & $\begin{array}{l}\text { One benign (1) CNV of chromosome } 14 \text { (q11.2) } \\
\text { Multiple LOH/AOH of many chromosomes }\end{array}$ \\
\hline 84 & 3p21.31-p21.1 & 7 & 34 & No CNVs; multiple LOH/AOH of many chromosomes \\
\hline 85 & $8 \mathrm{q} 23.1 \mathrm{q} 23.2 \mathrm{q} 23.3$ & 6 & 14 & $\begin{array}{l}\text { No CNVs } \\
\text { No other LOH/AOH }\end{array}$ \\
\hline 87 & $6 \mathrm{q} 21 \mathrm{q} 22.1 \mathrm{q} 22.2 \mathrm{q} 22.31$ & 12 & 60 & $\begin{array}{l}\text { No CNVs } \\
\text { One LOH/AOH of chromosome } 11\end{array}$ \\
\hline 101 & $6 \mathrm{q} 14.1-\mathrm{q} 15$ & 10.5 & 51 & One benign CNV \\
\hline \multirow{8}{*}{105} & 1q23.1-q31.1 & 29.5 & 232 & \multirow{8}{*}{$\begin{array}{l}\text { Multiple benign/likely benign CNVs } \\
\text { Multiple LOH/AOH of many chromosomes }\end{array}$} \\
\hline & $3 q 27.1-q 29$ & 13.6 & 108 & \\
\hline & $5 q 33.3-q 35.3$ & 24.7 & 195 & \\
\hline & $7 \mathrm{p} 14.2 \mathrm{p} 14.1$ & 5.3 & 22 & \\
\hline & $10 \mathrm{p} 15.1 \mathrm{p} 14$ & 5.4 & 34 & \\
\hline & $16 \mathrm{q} 22.3-\mathrm{q} 23.3$ & 10 & 44 & \\
\hline & 17p13.1-p11.2 & 11.7 & 124 & \\
\hline & $18 \mathrm{q} 12.1-\mathrm{q} 12.3$ & 13 & 46 & \\
\hline \multirow{2}{*}{112} & $5 \mathrm{p} 15.2 \mathrm{p} 15.1$ & 7 & 18 & One benign $\mathrm{CNV}$ \\
\hline & $9 q 31.1-q 32$ & 8.3 & 71 & $\mathrm{LOH} / \mathrm{AOH}$ of chromosome 11 (p11.2 p11.12) \\
\hline \multirow{12}{*}{169} & 1p13.3-p12 & 9.9 & 101 & \multirow{12}{*}{$\begin{array}{l}2 \text { likely benign CNVs of chromosomes } 8 \text { and } \mathrm{X} \\
\text { Multiple LOH/AOH of many chromosomes }\end{array}$} \\
\hline & $5 q 35.1-q 35.3$ & 8 & 107 & \\
\hline & $10 \mathrm{q} 11.21 \mathrm{q} 11.22$ & 5.4 & 49 & \\
\hline & $10 \mathrm{q} 21.1-\mathrm{q} 22.1$ & 19 & 81 & \\
\hline & $10 \mathrm{q} 24.1-\mathrm{q} 25.2$ & 14 & 133 & \\
\hline & $12 \mathrm{q} 22-\mathrm{q} 24.32$ & 34.3 & 281 & \\
\hline & $14 \mathrm{q} 23.3-\mathrm{q} 32.2$ & 30.3 & 231 & \\
\hline & 19q13.32-q13.42 & 7.2 & 272 & \\
\hline & 20q13.13-q13.32 & 8 & 43 & \\
\hline & 22q11.1-q12.1 & 11.4 & 173 & \\
\hline & Xp22.32-p21.1 & 27 & 123 & \\
\hline & $\mathrm{Xq} 23$ q24 & 9.8 & 51 & \\
\hline
\end{tabular}


TABLE 6: Continued.

\begin{tabular}{|c|c|c|c|c|}
\hline$\overline{\mathrm{DBN}}$ & Cytoband & Size $(\mathrm{mb})$ & Gene number & Remarks (associated CNVs and LOH/AOH) \\
\hline \multirow{2}{*}{178} & $5 \mathrm{p} 13.3 \mathrm{p} 13.2$ & 5.8 & 37 & No CNV \\
\hline & 17p11.2-q11.2 & 9.9 & 125 & No other $\mathrm{LOH} / \mathrm{AOH}$ \\
\hline \multirow{6}{*}{182} & $13 q 14.2-q 21.32$ & 18.6 & 75 & \multirow{6}{*}{$\begin{array}{l}\text { No CNV } \\
\text { No other } \mathrm{LOH} / \mathrm{AOH}\end{array}$} \\
\hline & $6 \mathrm{p} 22.3-\mathrm{p} 21.33$ & 10.3 & 269 & \\
\hline & $2 q 32.3 q 33.1$ & 5.2 & 717 & \\
\hline & $2 q 34-q 36.1$ & 9.6 & 765 & \\
\hline & $2 q 36.1-q 37.1$ & 7.4 & 622 & \\
\hline & $7 \mathrm{p} 22.2-\mathrm{p} 21.3$ & 5.7 & 49 & \\
\hline 189 & $17 q 21.32-q 23.2$ & 13.3 & 175 & No CNV \\
\hline \multirow{6}{*}{200} & $4 \mathrm{q} 32.3-\mathrm{q} 34.3$ & 9.7 & 616 & \multirow{6}{*}{$\begin{array}{l}\text { No CNV } \\
2 \text { other } \mathrm{LOH} / \mathrm{AOH}\end{array}$} \\
\hline & 2q11.1-q12.1 & 8.8 & 996 & \\
\hline & 2p12-p11.2 & 8.2 & 844 & \\
\hline & $2 \mathrm{p} 22.3 \mathrm{p} 22.2 \mathrm{p} 22.1$ & 6.1 & 719 & \\
\hline & 3 p26.1 p25.3 p 25.2 p 25.1 & 7.8 & 830 & \\
\hline & $15 q 23-q 24.3$ & 8 & 1205 & \\
\hline \multirow{3}{*}{204} & $3 q 11.2$ q12.1 q12.2 q12.3 & 6 & 49 & \multirow{3}{*}{$\begin{array}{l}\text { One likely benign CNV } \\
\text { No other LOH/AOH }\end{array}$} \\
\hline & 16 p12.1 p11.2 p11.1 & 8 & 107 & \\
\hline & $16 \mathrm{q} 11.2 \mathrm{q} 12.1 \mathrm{q} 12.2$ & 7 & 40 & \\
\hline \multirow{2}{*}{211} & $5 q 21.3-q 22.3$ & 7.3 & 30 & No CNV \\
\hline & $14 \mathrm{q} 22.1-\mathrm{q} 23.1$ & 7.3 & 51 & One LOH/AOH of chromosome 7 \\
\hline \multirow{2}{*}{214} & 18q11.2-q12.1 & 5.6 & 29 & One likely benign $\mathrm{CNV}$ \\
\hline & 6 p25.2-p24.3 & 5.5 & 42 & Two LOH/AOH of chromosomes 12 and 19 \\
\hline 228 & $8 \mathrm{q} 24.21 \mathrm{q} 24.22$ & 5.5 & 20 & No CNV or no other LOH/AOH \\
\hline
\end{tabular}

${ }^{*}$ To investigate its clinical importance/significance/associations/and so forth there is a need for further study using SNP microarray of parents. DBN: database number.

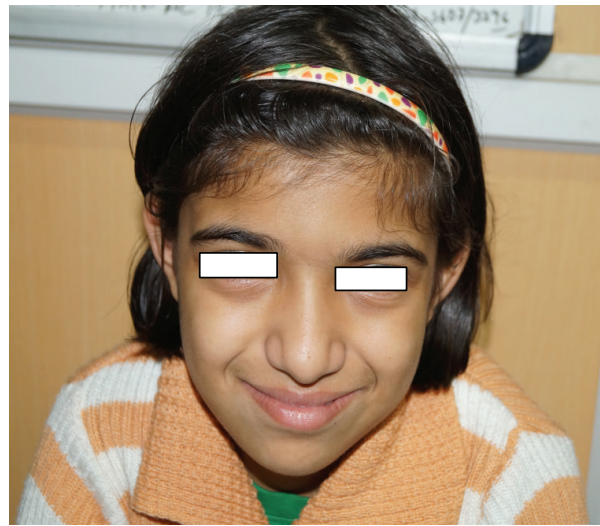

(a)

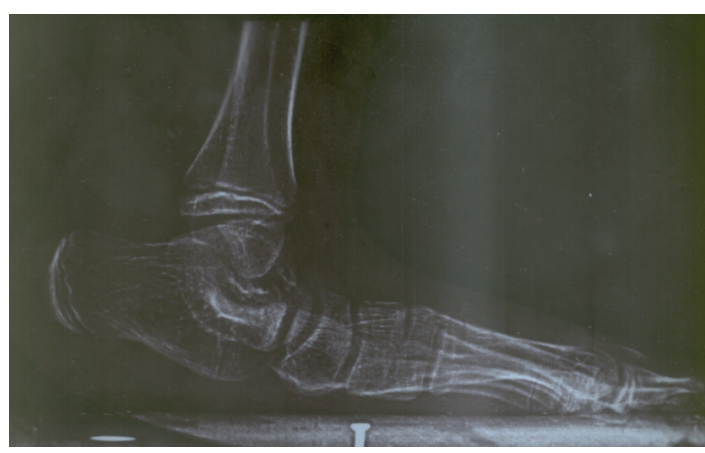

(c)

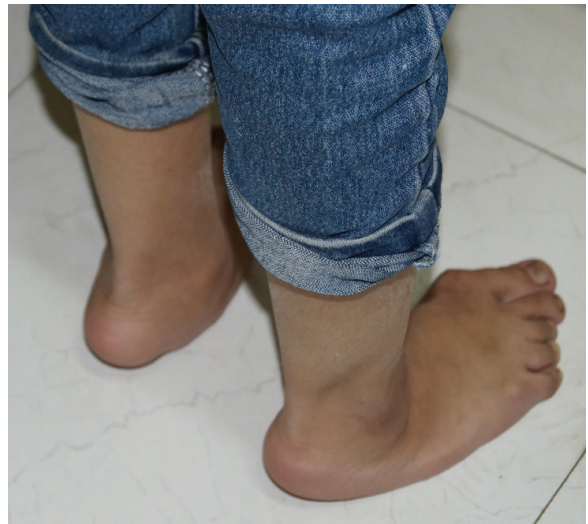

(b)

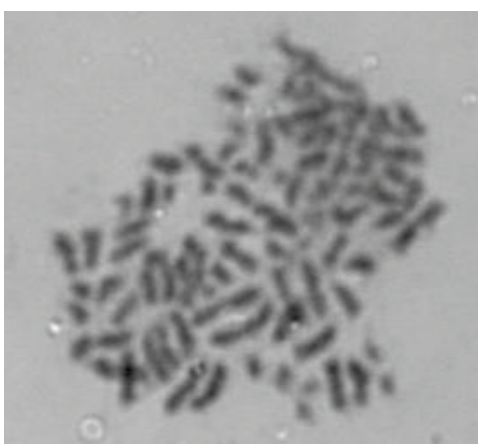

(d)

Figure 1: Mosaic triploidy case showing facial profile (a), flat rocker bottom foot ((b) clinical and (c) X-ray), and triploid metaphase cell (d). 


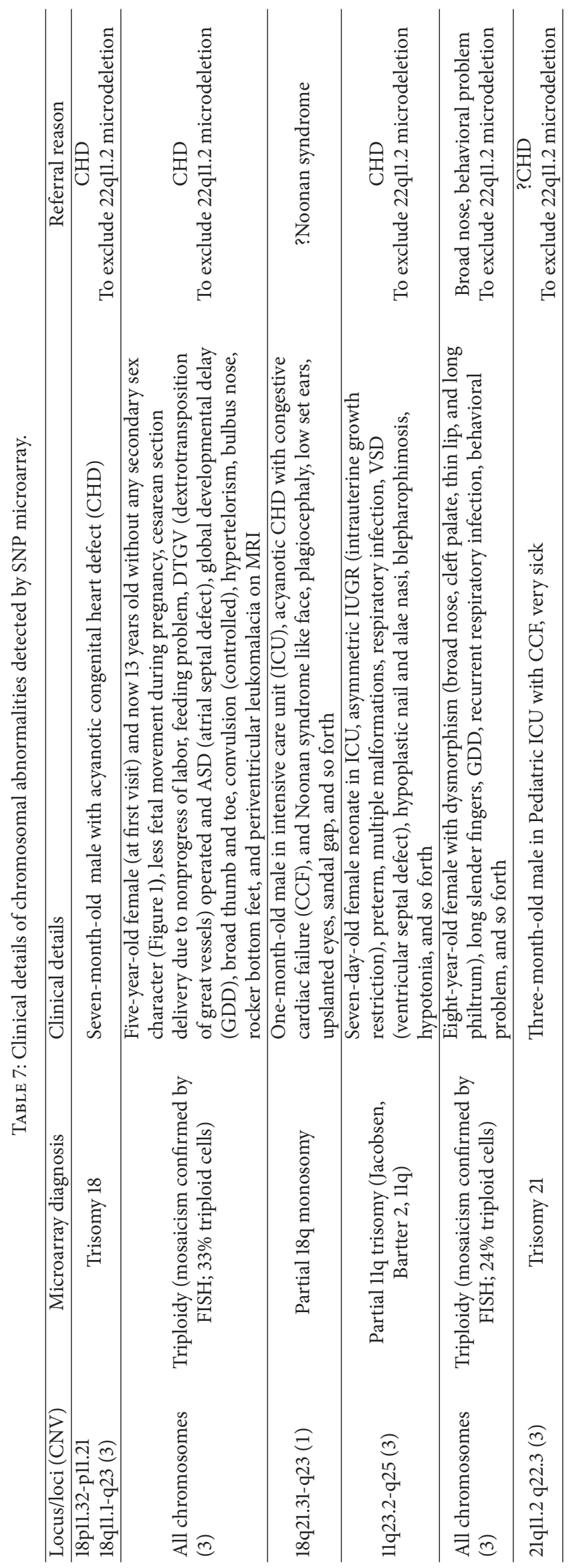




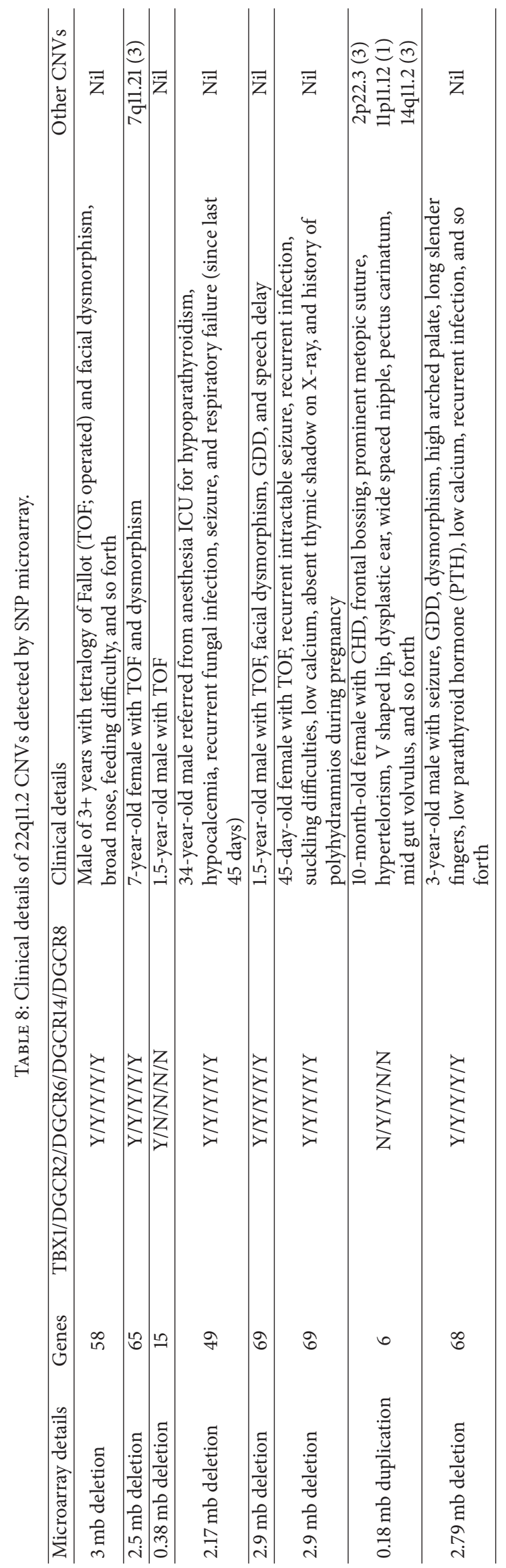


TABLE 9: Clinical details of other pathogenic CNVs detected by SNP microarray.

\begin{tabular}{|c|c|c|c|c|}
\hline Microarray details & Genes & Diagnosis & Clinical details & Other CNVs \\
\hline $\begin{array}{l}15 \mathrm{q} 11.2 \\
0.46 \mathrm{mb} \text { duplication }\end{array}$ & 6 & 15q11-q13 duplication & $\begin{array}{l}\text { Three-month-old male with TOF, feeding } \\
\text { difficulty, hypocalcemia, dysmorphism, } \\
\text { poly/syndactyle, hypoplastic mandible, } \\
\text { IUGR, and so forth }\end{array}$ & 10q11.22 (3) \\
\hline $\begin{array}{l}16 \mathrm{p} 11.2 \\
0.68 \mathrm{mb} \text { deletion }\end{array}$ & 46 & 16p11.2 deletion & $\begin{array}{l}\text { 4-month-male with CHD (DORV, } \\
\text { PS/pulmonary stenosis), } \\
\text { blepharophimosis, ptosis, and so forth }\end{array}$ & $\begin{array}{l}15 \mathrm{q} 12(3) \\
14 \mathrm{q} 11.2(3)\end{array}$ \\
\hline $\begin{array}{l}\text { 1q21.1 } \\
1.9 \mathrm{mb} \text { duplication }\end{array}$ & 24 & 1q21.1 duplication & $\begin{array}{l}\text { Male of } 2+\text { years with TOF, broad nose, } \\
\text { thin upper lip, absent philtrum, small and } \\
\text { low set ears, antimongoloid slant, } \\
\text { telecanthus, long slender fingers, widow } \\
\text { peak, and so forth }\end{array}$ & $11 p 11.12(3)$ \\
\hline $\begin{array}{l}15 \mathrm{q} 11.1 \mathrm{q} 11.2 \\
2.3 \mathrm{mb} \text { duplication }\end{array}$ & 13 & 15q11.1 q11.2 duplication & $\begin{array}{l}\text { 1-year-old male with CHD (VSD), } \\
\text { dysmorphism, GDD, and so forth }\end{array}$ & 14q11.2 (3) \\
\hline
\end{tabular}

TABLE 10: Clinical details of likely pathogenic CNVs detected by SNP microarray.

\begin{tabular}{|c|c|c|c|}
\hline Locus/loci & Microarray details & Genes & Clinical details \\
\hline $\begin{array}{l}22 \mathrm{q} 11.22 \\
7 \mathrm{q} 11.21\end{array}$ & $\begin{array}{l}0.4 \mathrm{mb} \text { duplication } \\
0.49 \mathrm{mb} \text { deletion }\end{array}$ & $\begin{array}{l}7 \\
3\end{array}$ & 2-year-old male with TOF \\
\hline $1 \mathrm{q} 21.2$ & $1.9 \mathrm{mb}$ duplication & 18 & 9-year-old male with TOF (operated) \\
\hline $\begin{array}{l}19 \mathrm{p} 13.11-12 \\
11 \mathrm{p} 11.2\end{array}$ & $\begin{array}{l}\text { 4.6 mb duplication } \\
3.1 \mathrm{mb} \text { duplication }\end{array}$ & $\begin{array}{l}35 \\
10\end{array}$ & 17-year-old female with TOF (operated) \\
\hline $\begin{array}{l}\mathrm{lq} 31.3 \\
\mathrm{Xq} 21.1-24 \\
\mathrm{Xq} 27.2-28\end{array}$ & $\begin{array}{l}3.8 \mathrm{mb} \text { triplication } \\
23.8 \mathrm{mb} \text { duplication } \\
6.7 \mathrm{mb} \text { duplication }\end{array}$ & $\begin{array}{l}13 \\
27 \\
18\end{array}$ & 13-year-old male with TOF (operated) \\
\hline $\begin{array}{l}\mathrm{lq} 21.1 \\
\mathrm{Xp} 22.33 \\
\end{array}$ & $\begin{array}{l}1.3 \mathrm{mb} \text { duplication } \\
1.2 \mathrm{mb} \text { duplication }\end{array}$ & $\begin{array}{l}12 \\
10\end{array}$ & 4-year-old male with TOF (operated) \\
\hline $\begin{array}{l}15 \mathrm{q} 11.2 \\
10 \mathrm{q} 11.22\end{array}$ & $\begin{array}{l}2.2 \mathrm{mb} \text { deletion } \\
2.1 \mathrm{mb} \text { duplication }\end{array}$ & $\begin{array}{l}19 \\
23\end{array}$ & $\begin{array}{l}\text { One-month-old male with hypocalcaemia } \\
\text { (Ca 5.6; PTH-46), convulsion, osteopenia, } \\
\text { squint, small toe, deep furrow feet, and so } \\
\text { forth (Figure 2) }\end{array}$ \\
\hline $22 \mathrm{q} 11.23$ & $0.26 \mathrm{mb}$ duplication & 2 & $\begin{array}{l}\text { 6-year-old female with dysmorphism, } \\
\text { square nose tip, proportionate short stature, } \\
\text { cyanotic CHD (tricuspid atresia, ostium } \\
\text { secundum ASD, etc.), clubbing, tracheal } \\
\text { shift, right pneumothorax with lung } \\
\text { collapse, right anotia, synophrys, pear } \\
\text { shaped nose, webbed neck, and so forth }\end{array}$ \\
\hline
\end{tabular}

FISH, mostly from FISH failure samples (frozen/clotted samples; FISH not tried). In fact, it was unexpected from FISH to identify small atypical deletions outside the region of hybridization in two cases (database numbers 48 and 191), which were identified by microarray. Thus, microarray contributed actual improvement by $\sim 2 \%$ above FISH for specific 22q11.2 deletions. However, SNP microarray provided many other pathogenic/likely pathogenic microdeletions/duplications besides aneuploidy/partial aneuploidy, triploidy, UPD, and other disorders. We have also observed poor clinical criteria as the leading contributor of failure to detect specific 22q11.2 deletion. Variations in deletion size and/or break point difference (with genes involvement) as well as other CNVs with or without $\mathrm{LOH}$ were evident. This study also identified several cases of $\mathrm{LOH} / \mathrm{AOH}$ loci with known and well-defined UPD disorders. Several cases of suspected $\mathrm{LOH} / \mathrm{AOH}$ were also identified; however, confirmation of association needs additional investigation using SNP microarray/QF PCR of their parents before claiming causative.

One of the major referral criteria for our patients was congenital heart disease, mainly conotruncal heart defect [7]. Several studies have established the importance of $22 \mathrm{q} 11.2$ CNVs in the etiology of congenital heart disease (CHD) with or without other associated malformations $[14,15]$. Patients with 22q11.2 duplication also present clinical features like 22q11.2 deletion syndrome, including heart defect [16]. In 22q11.2 duplication syndrome, most prevalent heart defect was conotruncal heart defect [17]. This is also true in animal experiment where mice carrying extra copies of TBX1 gene 


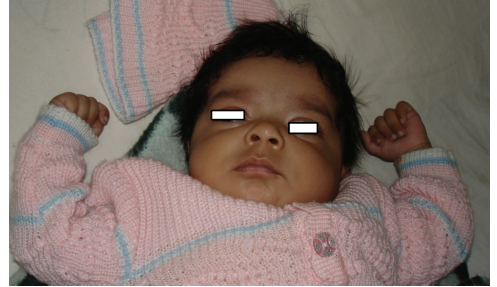

(a)

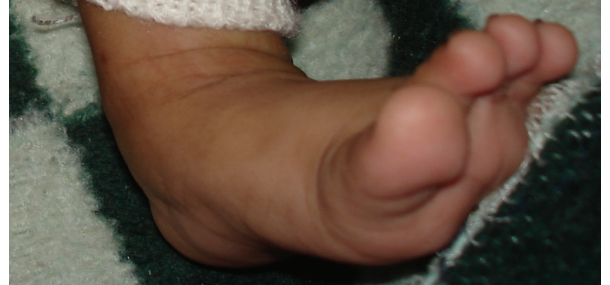

(b)

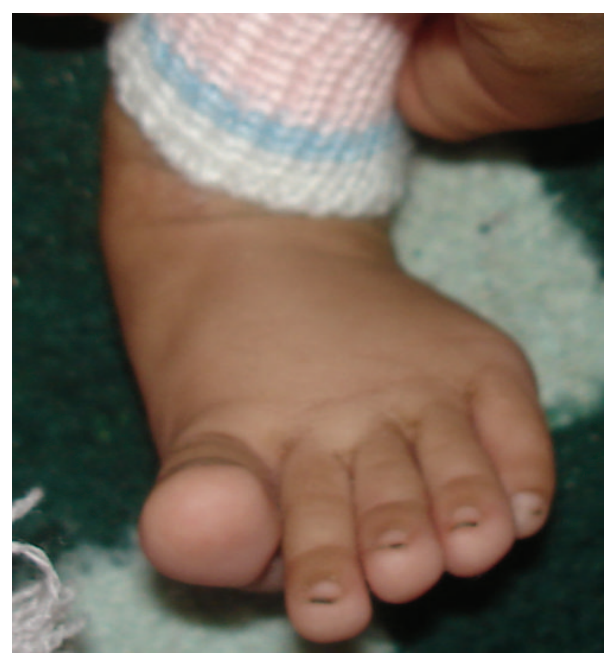

(c)

Figure 2: Male child with 15q11.2 deletion and 10q11.22 duplication showing squint, small toe and deep furrow feet.

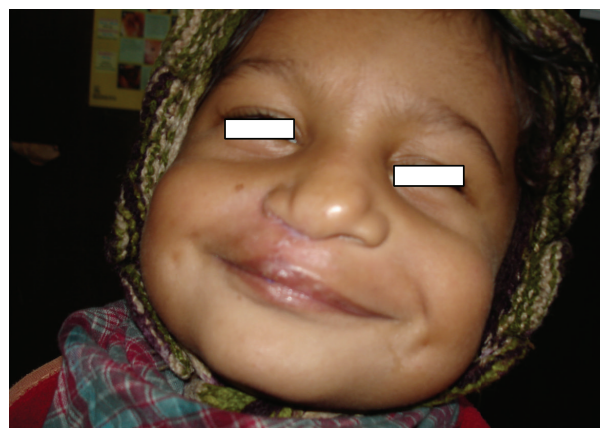

(a)

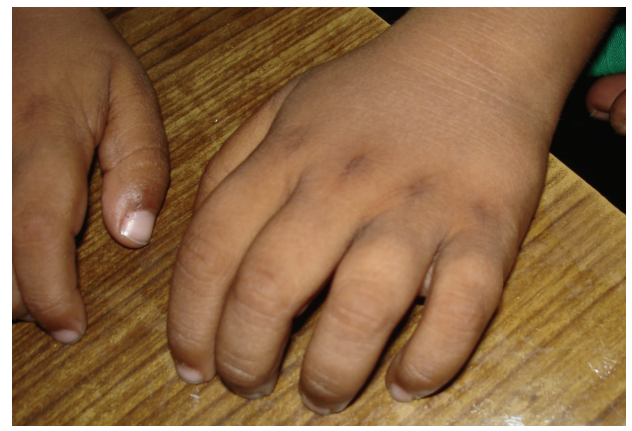

(b)

FIGURE 3: Male child with LOH/AOH of 6p22.3-p21.32 showing repaired cleft lip, broad and bifid nose, small left cornea and eye, long fingers, and rough creased skin over hands.

display full clinical picture of 22q11.2 deletion syndrome [18]. In this study we have found 2 cases of atypical small CNVs of 22q11.2 (duplication of $0.18 \mathrm{mb}$ spanning 18844632-19033532 and containing genes like DGCR6, PRODH, KIAA1647, DGCR9, DGCR10, and DGCR2 and deletion of $0.38 \mathrm{mb}$ spanning 19405537-19792353 and containing genes like UFD1L, PI4KA, SERPIND1, SNAP29, CRKL, AIFM3, AIFM3, LZTR1, THAP7, FLJ39582, MGC16703, P2RX6, P2RX6, SLC7A4, P2RX6P, LOC400891, and TBX1) with multiple malformations including cardiac malformations. These atypical small deletions are rarely reported $[13,19]$. The role of TBX1 in heart development has been already demonstrated in mice [20] and
TBX1 mutations have also been identified in individuals with TOF [21]. We have also observed TBX1 deletion with TOF, in our series.

This study detected several cases of $\mathrm{LOH} / \mathrm{AOH}$ of known UPD disorders (Table 5) and some more likely pathogenic UPDs (Table 6); however, further study using SNP microarray on parental DNA is required before reporting associations with specific developmental disorders.

Our study indicates that microarray should be first tier of test when samples are scant, lysed, or clumped/clotted/frozen as FISH or conventional cytogenetics are bound to fail/noninformative. This study suggests that microarray is a superior 


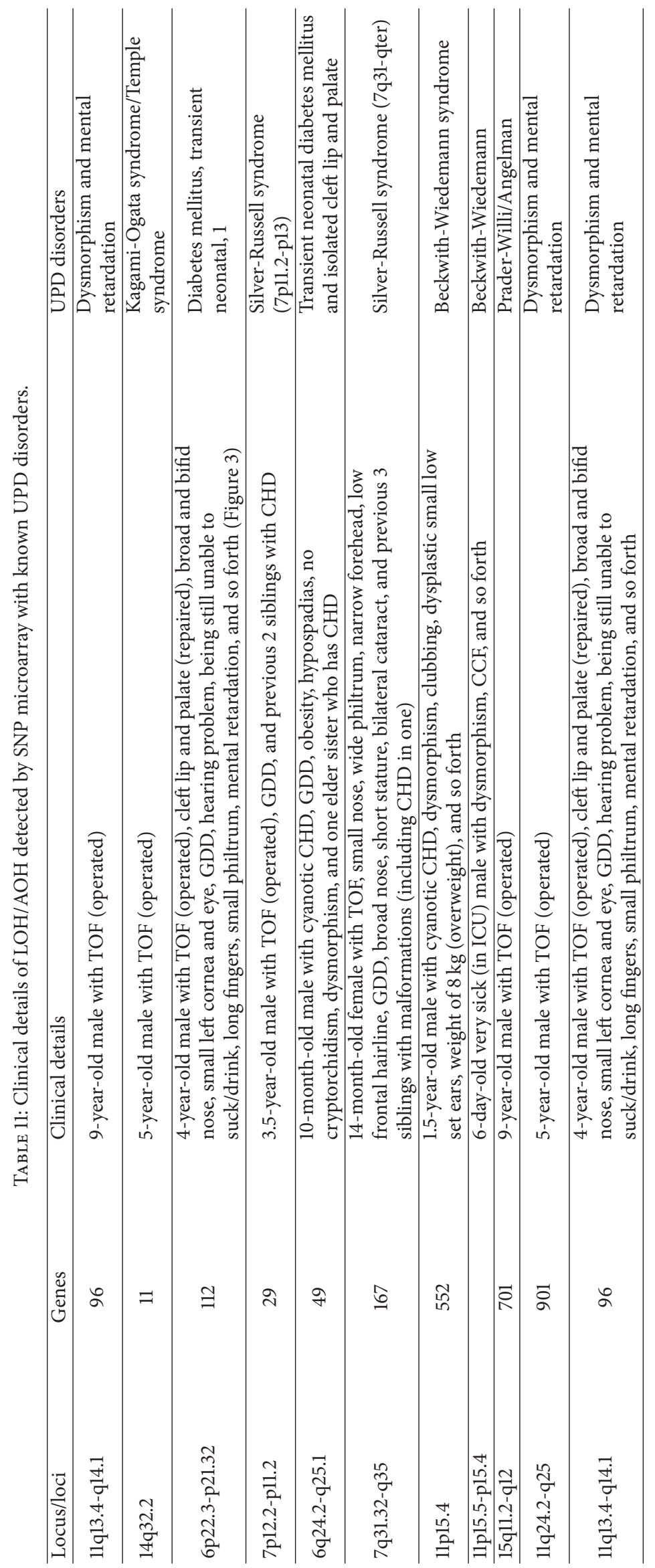


technique in clinically doubtful cases as well as ICU admissions on life support and 22q11.2 microdeletion is suspected on the basis of convulsion and/or cardiac defect/failure. Furthermore, in early weeks of life dysmorphology and malformation detection is difficult, in particular sick neonate on life support. We have observed in this study that clinically suspected microdeletion syndrome cases are frequently associated with second/more hits (deletion or duplication) elsewhere in the genome (tables/associated CNVs). Microarray also detected several cases of chromosomal aneuploidy, partial aneuploidy, triploidy, and so forth. This approach of DNA microarray will provide the highest chance of making a diagnosis and sparing the patient unnecessary diagnostic testing from many places, in addition to saving crucial times. Our conclusion is in agreement with the consensus statement [22].

We conclude that more strict clinical criteria should be followed for FISH test. If clinical diagnosis is uncertain or doubtful then microarray should be the first screening test. This is most important with newborn/neonate in intensive care unit as clinical criteria are few and difficult to elicit. Microarray is applicable in all samples irrespective of frozen, lysed, clotted, clumped, and other samples. Furthermore, SNP microarray provides information on aneuploidy, triploidy, partial aneuploidy, and associated small CNVs (often many) besides information on $\mathrm{LOH} / \mathrm{AOH}$ (indicating UPD disorders/in case of consanguinity homozygosity of recessive disorders). FISH may be used for detecting mosaicism, screening family members, prenatal diagnosis, and preimplantation diagnosis of specific deletions in proven family.

\section{Competing Interests}

The authors declare that there are no competing interests regarding the publication of this paper.

\section{Acknowledgments}

The study was supported by grant from Indian Council of Medical Research, New Delhi, India. Authors thank Departments of Pediatrics, Pediatric Surgery, Cardiovascular Surgery, and Cardiology of All India Institute of Medical Sciences and various hospitals of Delhi, India, for referring the cases for the research/service, and also they thank the family members of patients for their cooperation during the study. Authors acknowledge Professor Mariano Rocchi (University of Bari, Italy) for providing molecular probes (PAC/BAC clones) for FISH study.

\section{References}

[1] S. Óskarsdóttir, M. Vujic, and A. Fasth, "Incidence and prevalence of the 22q11 deletion syndrome: a population-based study in Western Sweden," Archives of Disease in Childhood, vol. 89, no. 2, pp. 148-151, 2004.

[2] K. Devriendt, J.-P. Fryns, G. Mortier, M.-N. Van Thienen, and K. Keymolen, "The annual incidence of DiGeorge/velocardiofacial syndrome," Journal of Medical Genetics, vol. 35, no. 9, pp. 789790, 1998.
[3] C. Cancrini, P. Puliafito, M. C. Digilio et al., "Clinical features and follow-up in patients with 22q11.2 deletion syndrome," Journal of Pediatrics, vol. 164, no. 6, pp. 1475-e2, 2014.

[4] A. K. Ryan, J. A. Goodship, D. I. Wilson et al., "Spectrum of clinical features associated with interstitial chromosome 22q11 deletions: a European collaborative study," Journal of Medical Genetics, vol. 34, no. 10, pp. 798-804, 1997.

[5] A. Halder, A. Fauzdar, M. Kabra, and A. Saxena, "Detection of 22q11.2 hemizygous deletion by interphase FISH in a patient with features of CATCH22 syndrome," Indian Pediatrics, vol. 42, no. 12, pp. 1236-1239, 2005.

[6] A. Halder, M. Jain, M. Kabra, and N. Gupta, "Mosaic 22q11.2 microdeletion syndrome: diagnosis and clinical manifestations of two cases," Molecular Cytogenetics, vol. 1, no. 1, p. 18, 2008.

[7] A. Halder, M. Jain, I. Chaudhary, and M. Kabra, "Prevalence of 22q11.2 microdeletion in 121 patients with cardiac malformation," BMC Medical Genetics, vol. 11, article 101, 2010.

[8] A. Halder, M. Jain, I. Chaudhary, N. Gupta, and M. Kabra, "Fluorescence in-situ hybridization (FISH) using non-commercial probes in the diagnosis of clinically suspected microdeletion syndromes: an experience with 301 cases," Indian Journal of Medical Research, vol. 138, pp. 135-142, 2013.

[9] J. D. Weisfeld-Adams, L. Edelmann, I. K. Gadi, and L. Mehta, "Phenotypic heterogeneity in a family with a small atypical microduplication of chromosome 22q11.2 involving TBX1," European Journal of Medical Genetics, vol. 55, no. 12, pp. 732736, 2012.

[10] M. Chen, Y.-S. Yang, J.-C. Shih et al., "Microdeletions/duplications involving TBX1 gene in fetuses with conotruncal heart defects which are negative for 22q11.2 deletion on fluorescence in-situ hybridization," Ultrasound in Obstetrics and Gynecology, vol. 43, no. 4, pp. 396-403, 2014.

[11] A. Halder, M. Jain, I. Chaudhary, and B. Varma, "Chromosome 22q11.2 microdeletion in monozygotic twins with discordant phenotype and deletion size," Molecular Cytogenetics, vol. 5, article 13, 2012.

[12] M. Manning and L. Hudgins, "Array-based technology and recommendations for utilization in medical genetics practice for detection of chromosomal abnormalities," Genetics in Medicine, vol. 12, no. 11, pp. 742-745, 2010.

[13] C. Poirsier, J. Besseau-Ayasse, C. Schluth-Bolard et al., "A French multicenter study of over 700 patients with 22q11 deletions diagnosed using FISH or aCGH," European Journal of Human Genetics, 2015.

[14] J. Breckpot, B. Thienpont, Y. Arens et al., "Challenges of interpreting copy number variation in syndromic and nonsyndromic congenital heart defects," Cytogenetic and Genome Research, vol. 135, no. 3-4, pp. 251-259, 2011.

[15] D. Warburton, M. Ronemus, J. Kline et al., “The contribution of de novo and rare inherited copy number changes to congenital heart disease in an unselected sample of children with conotruncal defects or hypoplastic left heart disease," Human Genetics, vol. 133, no. 1, pp. 11-27, 2014.

[16] M.-F. Portnoï, "Microduplication 22q11.2: a new chromosomal syndrome," European Journal of Medical Genetics, vol. 52, no. 23, pp. 88-93, 2009.

[17] R. F. M. Rosa, P. R. G. Zen, C. P. Ricachinevsky et al., "22q11.2 duplication and congenital heart defects," Arquivos Brasileiros de Cardiologia, vol. 93, no. 4, pp. e55-e57, 2009.

[18] J. Liao, L. Kochilas, S. Nowotschin et al., "Full spectrum of malformations in velo-cardio-facial syndrome/DiGeorge 
syndrome mouse models by altering Tbxl dosage," Human Molecular Genetics, vol. 13, no. 15, pp. 1577-1585, 2004.

[19] DECIPHER, https://decipher.sanger.ac.uk/search? q=22\%3A18844632$19033532+\#$ consented-patients/results.

[20] L. A. Jerome and V. E. Papaioannou, "DiGeorge syndrome phenotype in mice mutant for the T-box gene, Tbxl," Nature Genetics, vol. 27, no. 3, pp. 286-291, 2001.

[21] R. Rauch, M. Hofbeck, C. Zweier et al., "Comprehensive genotype-phenotype analysis in 230 patients with tetralogy of Fallot," Journal of Medical Genetics, vol. 47, no. 5, pp. 321-331, 2010.

[22] D. T. Miller, M. P. Adam, S. Aradhya et al., "Consensus statement: chromosomal microarray is a first-tier clinical diagnostic test for individuals with developmental disabilities or congenital anomalies," The American Journal of Human Genetics, vol. 86, no. 5, pp. 749-764, 2010. 

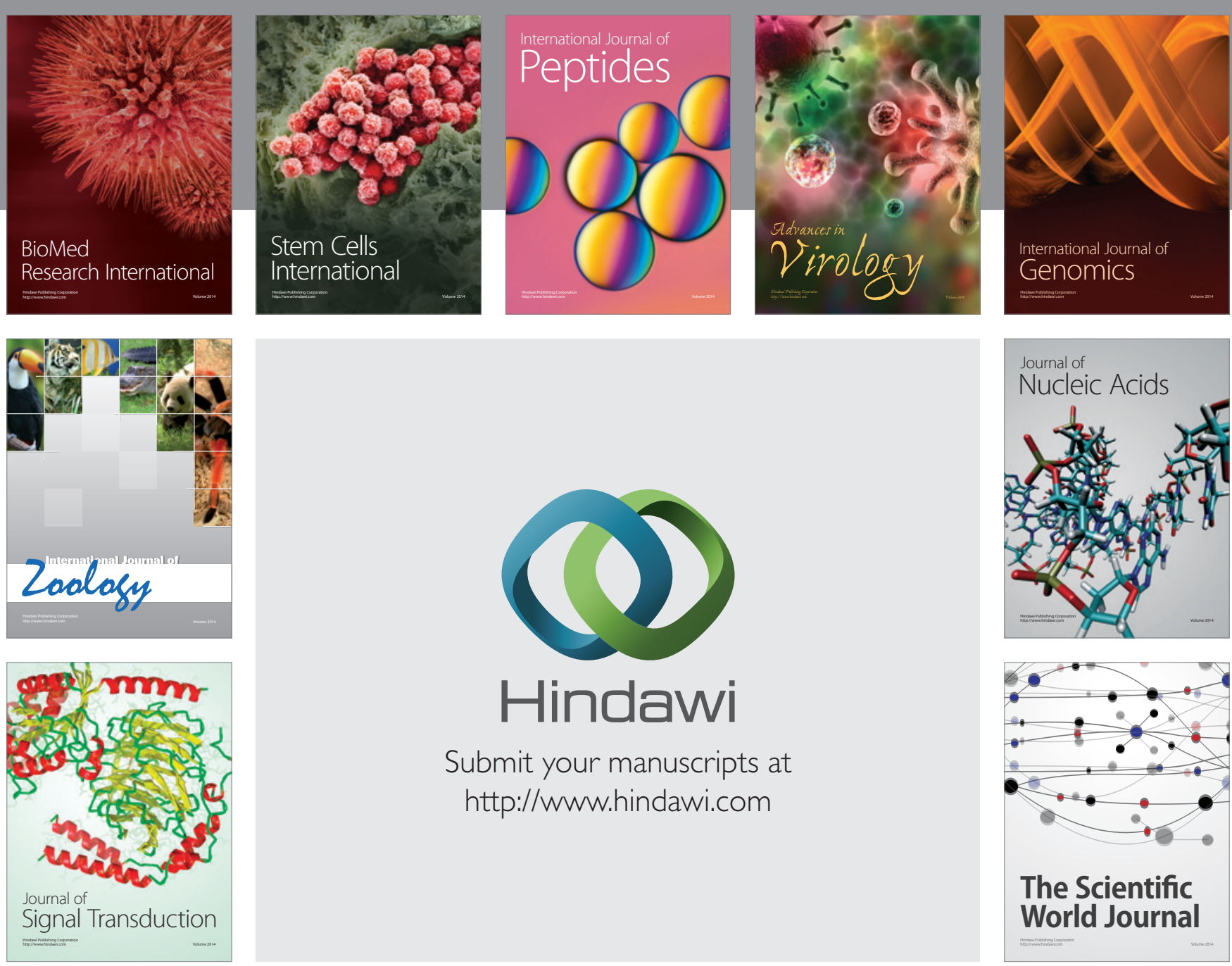

Submit your manuscripts at

http://www.hindawi.com
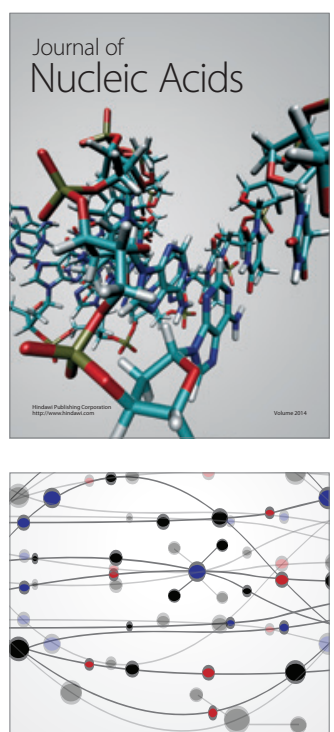

The Scientific World Journal
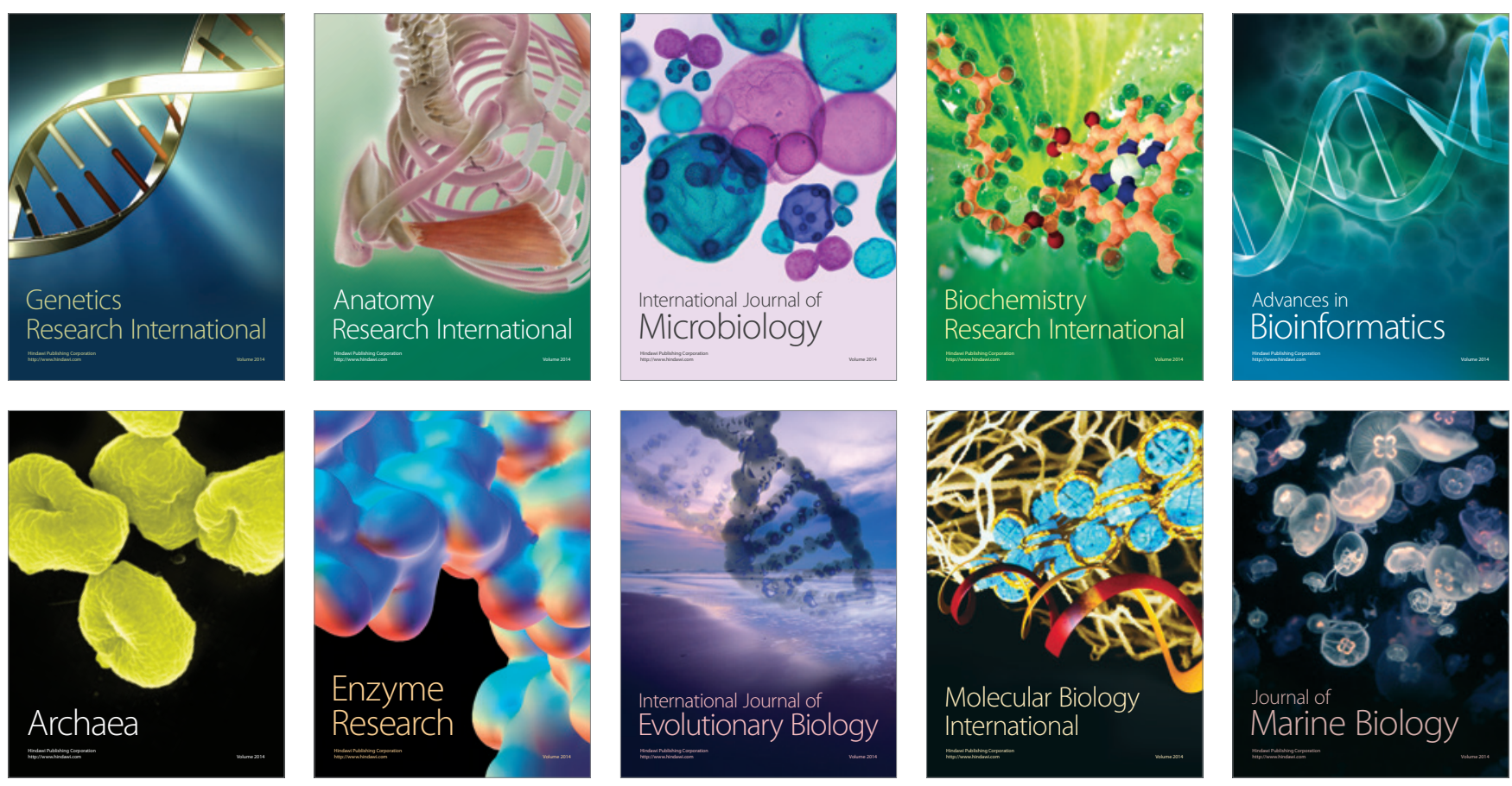